\title{
Acquisition Method of Three Dimensional Images Based on Liquid Crystal Micro Lens Array
}

\author{
Fan PAN ${ }^{1}$, Hui LI ${ }^{2,3 *}$ \\ ${ }^{1}$ College of Post and Telecommunication, Wuhan Institute of Technology, Wuhan 40073, P. R. China \\ ${ }^{2}$ School of Computer Science and Engineering, Wuhan Institute of Technology, Wuhan 40073, P. R. China \\ ${ }^{3}$ Hubei Key Laboratory of Intelligent Robot, Wuhan 40073, P. R. China \\ crossref http://dx.doi.org/10.5755/j01.ms.23.4.16677
}

Received 05 November 2016; accepted 31 March 2017

\begin{abstract}
This paper presents a novel method for acquiring three-dimensional (3D) images based on an electrically controlled liquid crystal (LC) micro lens array (MLA). The proposed method is extremely different from existing 3D imaging methods, because adapting the proposed LC-MLA as a key element for a 3D imaging system eliminates the conventional need for mechanical movements. The LC-MLA has $50 \mu \mathrm{m}$ hole-pattern electrodes, with an operating voltage as low as $0.2 \mathrm{Vrms}$, a focal length range of $50-400 \mu \mathrm{m}$, millisecond response time, and optical consistency between neighboring elements of $9 \%$. In order to demonstrate its 3D imaging capability, this paper describes an imaging experiment, in which two-dimensional (2D) images with different perspectives and magnifications are obtained by tuning the external applied voltage from 0.2 to $5.0 \mathrm{Vrms}$ at $0.5 \mathrm{Vrms} / \mathrm{step}$; then, a ray back projection algorithm is applied to process the 2D images in order to reconstruct the 3D image of the target object. This 3D imaging system is innovative, compact, and simple, making it very attractive for compact 3D imaging camera systems.

Keywords: liquid crystal device, micro-lens array, three dimensional imaging system.
\end{abstract}

\section{INTRODUCTION}

In recent years, the popular new technology of threedimensional (3D) imaging has swept the whole world through movies, TV shows, games, and medical image analysis. Generally, glass lenses or glass arrays constitute the key components of 3D optical imaging systems [1]. Traditional methods adopt an optical imaging lens or array as a key imaging element; these imaging systems require mechanical movements in order to focus and image, because the glass lens or glass array has a fixed focal length. As a result, imaging systems are generally bulky, heavy, and expensive. To overcome these limitations, the industry is badly in need of a novel 3D imaging system that does not require mechanical movement.

Liquid crystal (LC) is a classic birefringence material that can be applied in fabricating different kinds of optical devices, such as LC displays, LC lenses, LC microlens arrays, LC Fabry-Perot devices, LC filters, and LC optical switches. The most representative of these devices is a LC lens/array, which has electrically controlled tenable focal length, compactness, and portability; furthermore, it is easily integrated with other optical systems. At the current level of development, the LC lens/array can act as a substitute for some traditional optical lenses or arrays, to a certain extent [2,3]. Recently, the real 3D object got by LC lens is proposed [4]. But, there is still no studies from the angle of considering LC lens to be compound eyes for acquiring $3 \mathrm{D}$ images in a real $3 \mathrm{D}$ scene.

This paper introduces a novel 3D imaging system based on a design that incorporates an LC lens. Section 2 presents device fabrication and related theory for the

\footnotetext{
* Corresponding author. Tel.: +86-27-87992007; fax: +86-27-87992007

E-mail address: lihui00317@163.com (H. Li)
}

proposed LC lens. Some classical photoelectric features, measurement results, and interesting 3D imaging application of the LC lens are discussed and analyzed in Section 3. Finally, this paper offers conclusions about the novel 3D imaging system based on the proposed LC lens.

\section{DEVICE FABRICATION AND RELATED THEORY}
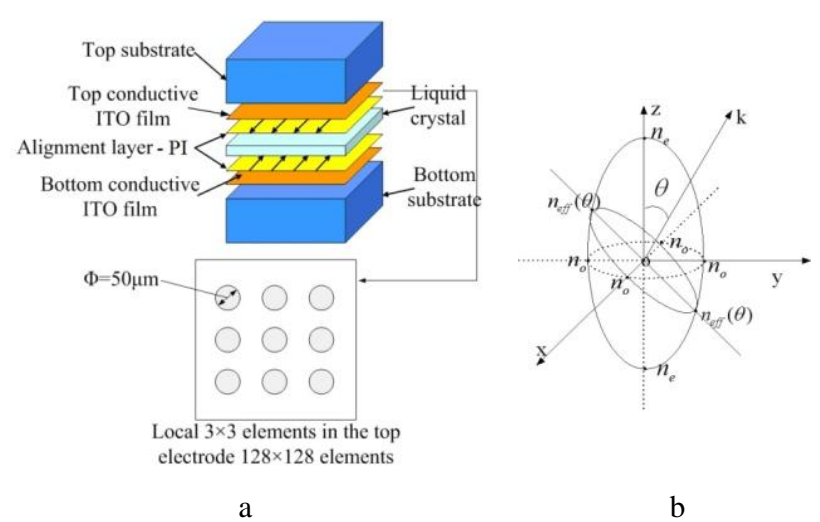

Fig. 1. Schematic diagram of LC lens: a-the LC lens structure schematic diagram, which represents a local $3 \times 3$ element in the top electrode $128 \times 128$ elements (That is only schematic local amplification region of the proposed array); $b$ - refractive index ellipsoid

Based on previous research [5-8], this paper proposes a new LC micro lens array (MLA) that can be fabricated simply and at low cost. The important improvement to the structure involves replacing the insulating glass with a polyimide (PI) layer, which can effectively lower the operating voltage, as shown in Fig. 1 a. The PI layers, which rub in a horizontal direction, also function as 
alignment layers. Lithography and hydrochloric acid are applied to form the top pattern-electrode array with $128 \times 128$ elements, which have $50 \mu \mathrm{m}$ diameters and $100 \mu \mathrm{m}$ of distance between neighboring elements (the distance between holes is measured from the edges of the adjacent holes). The thickness of the LC (E44, Merck) layer is $20 \mu \mathrm{m}$.

LC is a birefringence material. This property causes incident light with different polarizations to meet different refractive indices. The parallel beam, called ordinary light, encounters the same index consistently, regardless of the included angle between the polarization of the incident light and the optical axis, as shown in Fig. $1 \mathrm{~b}$. On the other hand, the polarization direction is perpendicular to the plane of the optical axis, called extraordinary light, sees a refractive index that is direction-dependent. The index varies by the included angles between the polarization of the incident light and the optical axis. The refractive index can be calculated as follows:

$n_{e f f}^{2}(\theta)=\frac{n_{o}^{2} n_{e}^{2}}{n_{e}^{2} \cos ^{2} \theta+n_{o}^{2} \sin ^{2} \theta}$,

where $\theta$ denotes the angle between the polarization of the incident light and the LC optical axis, and $n_{e}$ and $n_{o}$ represent, respectively, the refractive index for the extraordinary light beam and the ordinary light beam. The refractive index ellipsoid is shown in Fig. $1 \mathrm{~b}$.

We assembled a LC sample according to the fabrication flow described above. When there is no external applied voltage, all LC molecules have the same refractive index of $n_{e}$. With applied voltage, marginal LC molecules of $n_{o}$ rotate $90^{\circ}$ under the effect of the electric field. The refractive index of LC in the central area is still $n_{e}$, the same as the initial time. This creates a gradient distribution of the refractive index in the LC sample; this LC sample then produces the effect of a convex lens. Based on the geometrical optics and elastic continuum theory, the focal length formula of LC-MLA is as follows [6-8]:

$$
f=\frac{r^{2}}{2 \Delta n \cdot d_{L C}},
$$

where $r$ is the radius of aperture, $\Delta n$ is the LC birefringence difference, and $d_{\mathrm{LC}}$ is the thickness of the LC layer.

\section{EXPERIMENTS AND DISCUSSION}

An experiment was conducted to test the LC sample. The experimental setup for classic electro-optical properties of the proposed LC-MLA is shown in Fig. 2. The LC sample was set on an optical displacement platform between two polarizers. The light source used was a white parallel light. The light transmitted through the LC sample before falling on a Charge-coupled Device (CCD). The CCD was 3 megapixels, $1 / 2$ inch, and $6.4 \mathrm{~mm} \times 4.8 \mathrm{~mm}$ in size, and it was connected to a computer (PC). A function generator with tunable applied voltage was utilized to drive the LC sample.

The white light source was utilized in order to measure the focal length values of the proposed LC-MLA.

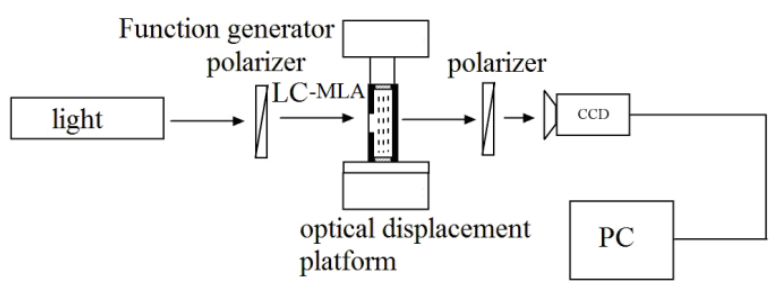

Fig. 2. Experimental setup

Throughout the whole measurement process, the strongest light points on the optical axis were the focal points. The corresponding focal length points were measured at different applied voltages. Finally, the focal length was plotted against the applied voltage, as shown in Fig. 3 a. The data indicate an inversely proportional relationship between these two values. The focal length changed along with the external applied voltage; as the value of the external applied voltage became much larger, the value of the focal length became much smaller. The range of values for focal length was tunable from $50 \mu \mathrm{m}$ to $400 \mu \mathrm{m}$, while the external applied voltage changed from 0.2 Vrms to 5.0 Vrms (The RMS presented here means the root mean square of voltage value).

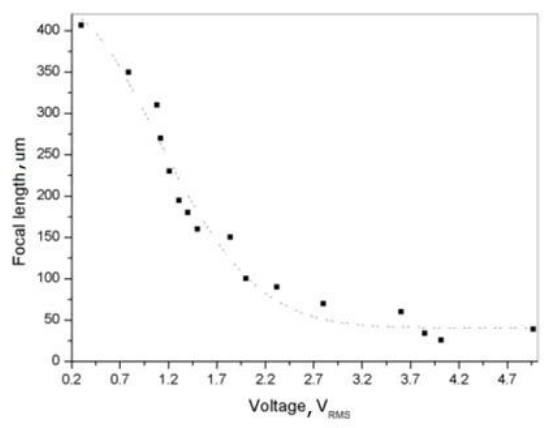

a
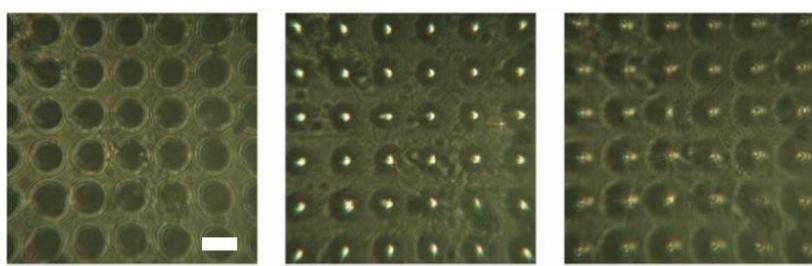

$0.2 \mathrm{Vmrs}$

$1.8 \mathrm{Vrms}$

$3.5 \mathrm{Vrms}$
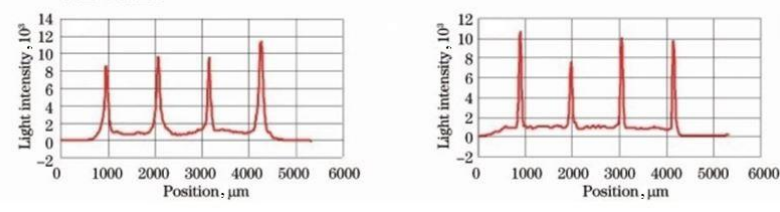

b

Fig. 3. The typical optical performance of the LC-MLA measured by white light: a-focal length versus applied voltage; $\mathrm{b}$-focusing procedure under different applied voltages, where the horizontal and vertical direction PSF (point of spread function) of the four elements is $1.8 \mathrm{Vrms}$, and scale bar of this figure is $50 \mu \mathrm{m}$

White parallel light was also utilized to examine the focusing ability of the proposed LC-MLA. The distance $(86 \mu \mathrm{m})$ between the LC-MLA and CCD was fixed, without any changes throughout the entire measurement 
process. Fig. $3 \mathrm{~b}$ shows different images obtained by the LC-MLA under different voltages. A voltage of $0.2 \mathrm{Vrms}$ produced a non-focused state, while a focused state occurred at $1.8 \mathrm{Vrms}$. Under these conditions, the point spread function (PSF) along the horizontal and vertical directions can be identified, and the optical consistency of neighboring elements is about $9 \%$. At $3.5 \mathrm{Vrms}$, the acquired image from CCD presents the state of defocusing. In this figure, the defocusing state of LC lens array is obviously presented. Compared the calculated values by Eq. 2 with those real measured values in Fig. $3 \mathrm{a}$, the theoretical values are consistent with the measured values. For example, the theoretical focal length is $78 \mu \mathrm{m}$ based on Eq. 2, and this is almost the same as the real measured value in Fig. 3 a. This demonstrates that the theory of geometrical optics and elastic continuum described above could effectively confirm that the proposed LC lens is a convex lens. And the design of the proposed LC lens could be also supported by the theory mentioned above.

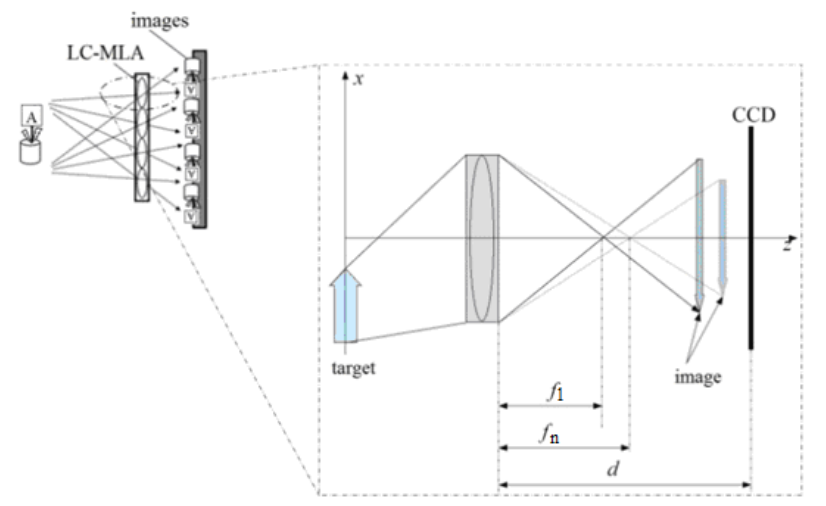

Fig. 4. Schematic diagram of 3D imaging system based on LCMLA

Fig. 4 illustrates a new type of 3D imaging system based on the proposed LC-MLA and a CCD. The proposed imaging system is relatively simple compared to conventional systems, which is its most important advantage. The current 3D system usually has so many glass optical lenses to acquire the $3 \mathrm{D}$ images. It is known that the conventional optical glass lens is generally expensive and bulk. The major simplified part in the proposed system is that the non-mechincal moving device LC-MLA is utilized. As it has a feature of electronically controlled focal length, the imaging system could be compact without relatively complicated multiple lens group structure compared to those conventional systems with some glass lenses. And the cost of building the system could be also reduced compared to those conventional system with some glass lenses. The target object was a piece of paper with the letter 'A' printed on it. Schematic experimental setup is presented in Fig. 4. The distance between the CCD and the LC-MLA was about $120 \mu \mathrm{m}$, and the target was set near the optical axis. Because the LC-MLA is a MLA, the transmitted light produced multiple $2 \mathrm{D}$ images with some very minor differences. In other words, the LC-MLA acquired a number of 2D images from different perspectives which is alike compound eyes; this represents the key factor underlying to construct a 3D imaging system. The specific operation process of the 3D imaging system involved changing the applied voltage at $0.5 \mathrm{Vrms} / \mathrm{step}$. The applied voltage changed within a range from 0.2 to $5.0 \mathrm{Vrms}$. The 2D images created an image stack, which could be utilized to reconstruct a $3 \mathrm{D}$ image. The magnification factor of the $i j^{\text {th }}$ element between the first image (taken at the shortest focal length) and the $n^{\text {th }}$ image is as follows:

$$
M_{i j n}=\frac{f_{1}\left(d-f_{n}\right)}{f_{n}\left(d-f_{n}\right)},
$$

where $f_{1}$ is the shortest focal length, $f_{\mathrm{n}}$ is the $n^{\text {th }}$ focal length, and $d$ is the distance between the LC-MLA and CCD.

Many methods exist for reconstructing 3D images of a target [9-12]. However, existing methods tend to be usually expensive and complicated. The method proposed in this paper is relatively simpler than conventional methods. This method utilizes a normal ray back projection algorithm and 2D images obtained by the LC-MLA in order to create 3D images. Because 2D images have different perspectives, a 3D image of the target can be reconstructed with the information provided by the LCMLA.
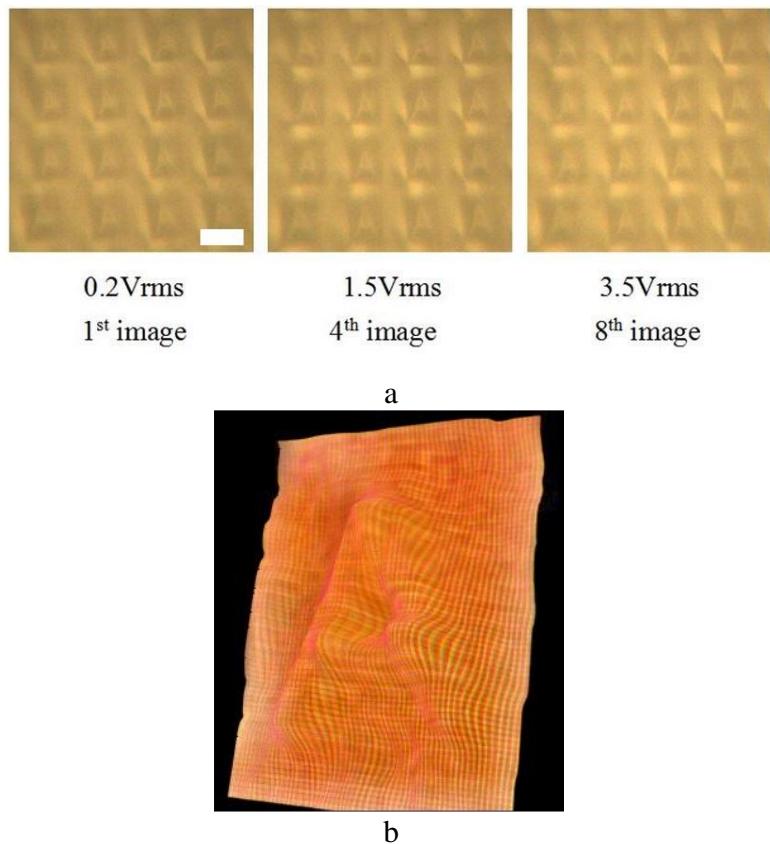

Fig. 5. Results of the 3D imaging system: a-considering spaces, the selected 2D images of the target object at voltages of $0.2 \mathrm{Vrms}, 1.5 \mathrm{Vrms}$, and $3.5 \mathrm{Vrms}$ have very slight differences in perspective and magnification, and scale bar of this figure is $50 \mu \mathrm{m} ; \mathrm{b}$-based on recorded $2 \mathrm{D}$ images, a 3D image of the target object can be reconstructed and represented with a $2 \mathrm{D}$ grid

The light intensity of each pixel in the reconstructed 3D image is as follows:

$I\left(x, y, z_{r}\right)=\frac{1}{M \times N \times k} \sum_{i=1}^{M} \sum_{j=1}^{N} \sum_{m=0}^{k-1} E\left[\frac{x}{M_{i j m \times\left(\frac{z_{r}}{z_{0}}\right)}}, \frac{y}{M_{i j m \times\left(\frac{z_{r}}{z_{0}}\right)}}, z_{r}\right]$, (4)

where $z_{\mathrm{r}}$ denotes the reconstructed plane, $M_{\mathrm{ijm}}$ is obtained through Eq. 3, $E_{\mathrm{m}}$ is the magnified image, $\mathrm{M} \times \mathrm{N}$ 
represents the $128 \times 128$ elements, and $\mathrm{k}$ is the total number of recorded $2 \mathrm{D}$ images.

Fig. $5 \mathrm{~b}$ shows the final reconstructed $3 \mathrm{D}$ image of the target object at $277 \times 317$ resolution. In order to present the final result clearly, we have already magnified the size of the final result in a certain extent. The image was acquired using Eq. 4. It was generated from back to front along the direction of the ray. The entire composition extends from the farthest place, $z_{\mathrm{r}}=400 \mu \mathrm{m}$, to the nearest place, $z_{\mathrm{r}}=50 \mu \mathrm{m}$, and the chosen step was about $10 \mu \mathrm{m} / \mathrm{step}$. This workflow direction ensures that masked parts of the target do not affect the resulting pixels. Fig. $5 \mathrm{~b}$ shows the $3 \mathrm{D}$ result, which has much more depth information compared to conventional 2D images. The letter ' $\mathrm{A}$ ' from the very beginning is not a 3D image. But, the letter ' $\mathrm{A}$ ' on a paper is in a real $3 \mathrm{D}$ scene. The proposed LC-MLA is alike a compound eyes. For every single element in the LC lens array, the target in the real $3 \mathrm{D}$ scene has a different two dimensional image. That is the same as the effect of the compound eyes. This imaging experiment is to propose a possible application in the field of $3 \mathrm{D}$ imaging. As it is known, this field contains so many topics, and it is so hot in recent years. The presented result is just a preliminary result of utilizing the proposed LCLMA in 3D imaging. And the result demonstrates a relatively strong sense of $3 \mathrm{D}$ imaging; however, the resolution is not high because of pixel and hardware limitations. This factor indicates room for improvement in the future.

\section{CONCLUSIONS}

This paper proposed a method for acquiring 3D images based on a LC-MLA, which is very different from conventional imaging methods. Using multiple 2D images under different perspectives and magnifications obtained by the proposed LC-MLA, the ray back projection algorithm can be successfully used to reconstruct a 3D image. The proposed 3D imaging system has potential applications in many imaging contexts, such as 3D TV, games, and movies. However, this is only a prototype with low resolution, and there is still a need for further refining of the design in the future.

\section{Acknowledgments}

This work was supported by a grant from National Natural Science Foundation of China (51703071), the Research Foundation of the Education Bureau of Hubei Province, China (B2016489, D20171504), the Science Foundation of the Wuhan Institute of Technology
(Q201601), and the Open Foundation for the Hubei Key Laboratory of Intelligent Robot (HBIR 201605).

\section{REFERENCES}

1. Stern, A., Javidi, B. Three-dimensional Image Sensing, Visualization, and Processing Using Integral Imaging Proceeding of the IEEE 94 (3) 2006: pp. 591-607.

2. Ren, H.W., Xu, S., Lin, Y.J., Wu, S.T. Adaptive Focus Lenses Optics and Photonics News 19 (10) 2008: pp. $42-47$. https://doi.org/10.1364/OPN.19.10.000042

3. Sato, S. Liquid-Crystal Lens-Cells with Variable Focal Length Japanese Journal of Applied Physics 18 (9) 1979: pp. $1679-1684$.

4. Hassanfiroozi, A., Huang, Y.P., Javidi, B., Shieh, H.P.D. Hexagonal Liquid Crystal Lens for 3D Endoscopy Optics Express 23 (2) 2015: pp. 971-981. https://doi.org/10.1364/OE.23.000971

5. Ye, M., Wang, B., Sato, S. Polarization Independent Liquid Crystal Lens with Four Liquid Crystal Layer IEEE Photonics Technnology. Letters 18 (3) 2006: pp. 505-507.

6. Ren, H.W., Wu, S.T. Adaptive Liquid Crystal Lens with Large Focal Length Tenablility Optics Express 14 (23) 2006: pp. $11292-11298$.

7. Love, G.D., Naumov, A.F. Modal Liquid Crystal Lenses Liquid Crystals Today 10 (1) 2000: pp. 1-4. https://doi.org/10.1080/135831401750061465

8. Chen, C.W., Cho, M., Huang, Y.P., Javidi, B. Three Dimensional Imaging with Axially Distributed Sensing Using Electronically Controlled Liquid Crystal Lens Optics Letters 37 (10) 2010: pp. 4125-4127.

9. Lee, Y.H., Peng, F., Wu, S.T. Fast-Response Switchable Lens for 3D and Wearable Display Optics Express 24 (2) 2016: pp. $1668-1675$.

10. Lee, C.K., Park, S.G., Monn, S., Lee, B. Viewing Zone Duplication of Multi-Projection 3D Displays System Using Uniaxial Crystal Optics Express 24 (8) 2016: pp. $8458-8470$. https://doi.org/10.1364/OE.24.008458

11. Tien, T.M., Sasaki, T., Sakamoto, M., Noda, K., Kawatsuki, N., Ono, H. Three-Dimensional Vector Holograms Formed in Twisted-Nematic Azo-Dye-Doped Polymer Liquid-Crystal Composite Journal of the Optical Society of America B 33 (2) 2016: pp. 286-291. https://doi.org/10.1364/JOSAB.33.000286

12. Hassanfiroozi, A., Huang, Y.P., Javidi, B., Shieh, H.P.D. Dual Layer Electrode Liquid Crystal Lens for 2D/3D Tunable Endoscopy Imaging System Optics Express 24 (8) 2016: pp. $8527-8538$. https://doi.org/10.1364/OE.24.008527 